\title{
Differential human brain activation by vertical and horizontal global visual textures
}

\author{
Jane E. Aspell • John Wattam-Bell • \\ Janette Atkinson • Oliver J. Braddick
}

Received: 10 August 2009 / Accepted: 18 January 2010 / Published online: 4 February 2010

(C) Springer-Verlag 2010

\begin{abstract}
Mid-level visual processes which integrate local orientation information for the detection of global structure can be investigated using global form stimuli of varying complexity. Several lines of evidence suggest that the identification of concentric and parallel organisations relies on different underlying neural substrates. The current study measured brain activation by concentric, horizontal parallel, and vertical parallel arrays of short line segments, compared to arrays of randomly oriented segments. Six subjects were scanned in a blocked design functional magnetic resonance imaging experiment. We compared percentage BOLD signal change during the concentric, horizontal and vertical blocks within early retinotopic areas, the fusiform face area and the lateral occipital complex. Unexpectedly, we found that vertical and horizontal parallel forms differentially activated visual cortical areas beyond V1, but in general, activations to concentric and parallel forms did not differ. Vertical patterns produced the highest percentage signal change overall and only area V3A showed a significant difference between concentric and parallel (horizontal) stimuli, with the former better activating this area. These data suggest that the difference in
\end{abstract}

J. E. Aspell ( $\square)$. O. J. Braddick

Department of Experimental Psychology,

University of Oxford, Oxford, UK

e-mail: jane.aspell@epfl.ch

J. Wattam-Bell · J. Atkinson

Department of Psychology, University College London,

London, UK

Present Address:

J. E. Aspell

Brain-Mind Institute, Ecole Polytechnique Fédérale de Lausanne,

SV 2805, Station 19, 1015 Lausanne, Switzerland brain activation to vertical and horizontal forms arises at intermediate or global levels of visual representation since the differential activity was found in mid-level retinotopic areas V2 and V3 but not in V1. This may explain why earlier studies-using methods that emphasised responses to local orientation-did not discover this vertical-horizontal anisotropy.

Keywords Global processing · Form vision · Form coherence $\cdot$ Orientation anisotropy $\cdot \mathrm{fMRI}$

\section{Introduction}

The analysis of a visual scene begins with the extraction of local contour information in early visual cortex. In order to correctly segment a scene and to enable complex object recognition, the local elements that are related and are likely to belong to a common cause (e.g. common contour, surface, texture etc.) must first be grouped together (Badcock and Clifford 2006). While decades of work has characterised the 'early' neural processing of local image features (Hubel and Wiesel 1968; Smith et al. 2002) as well as the 'later' processing of complex objects (Maunsell and Newsome 1987; Felleman and Van Essen 1991; Grill-Spector and Malach 2004), relatively little is known of the mechanisms of mid-level vision that bridge these stages. However, growing evidence indicates that the integration of local features into intermediately complex global forms involves processing in multiple sites in the primate brain, in both early retinotopic areas and higher areas in the ventral visual stream (Allman et al. 1985; Lamme et al. 1998; Achtman et al. 2003; Kourtzi et al. 2003; Grill-Spector and Malach 2004; Ostwald et al. 2008). It is likely that the integration of local features is driven by both bottom-up 
stimulus properties such as collinearity, proximity and connectedness (see e.g. Kofka 1935; Kovacs and Julesz 1993), as well as by top-down biases from stored object representations (Humphreys and Forde 2001).

As a consequence of the integration occurring in the larger receptive fields of neurons in extrastriate visual areas, more complex feature combinations can be encoded here than in primary visual cortex. A number of studies have shown that neurons in intermediate areas of the ventral stream, e.g. V2 and V4 (Gallant et al. 1993; Kobatake and Tanaka 1994; Mahon and De Valois 2001) are sensitive to the organization of global forms (concentric, radial and other complex shapes). The properties of V1, V2 and V4 cells have been characterised by single unit studies of macaque neurons using different types of forms or gratings (Gallant et al. 1993; Kobatake and Tanaka 1994; Mahon and De Valois 2001). Gallant et al. (1993) found that twice as many V4 neurons preferred non-Cartesian (concentric, hyperbolic and radial) to Cartesian (parallel) gratings. In a comparison of V1 and V2 neuronal responses, it was found that selectivity decreased for parallel and increased for nonCartesian patterns from V1 to V2 (Mahon and De Valois 2001). Evidence for the involvement of human V4 in concentric shape processing comes from a study of a patient with a $\mathrm{V} 4 \mathrm{v}$ lesion who had a deficit in concentric form processing (Gallant et al. 2000). In keeping with these results, a functional magnetic resonance imaging (fMRI) study reported that human V4 is activated more strongly by concentric gratings than by parallel gratings (Wilkinson et al. 2000). A more recent paper also reported greater fMRI activation to concentric Gabor array stimuli (Dumoulin and Hess 2007). Greater brain responses to concentric compared to translational Glass patterns were recently found in an event related potential study (Pei et al. 2005).

Many psychophysical studies (Maloney et al. 1987; Dakin 1997a, b, 1999; Wilson et al. 1997; Wilson and Wilkinson 1998, 2003; Kurki and Saarinen 2004; Lewis et al. 2004; Aspell et al. 2006; Bell et al. 2007) have investigated the mechanisms that mediate the integration of local features into global forms, and some have provided evidence for different mechanisms underlying the detection of concentric and parallel structure. These studies have used stimuli-Glass patterns, Gabor or simple line element displays - that generate the perception of a global form when their constituent elements (oriented dot pairs or Gabor/line elements) are organized as global configurations. Concentric and parallel global organization have some aspects in common-in that at certain scales concentric forms also have locally parallel texture-but concentric forms differ in a number of important ways, e.g., in being made up of curved contours and closed contours (Kovacs and Julesz 1993) and in having a more compelling global circular shape and symmetry. Comparisons of responses to these forms should therefore provide insight into the critical factors contributing to the global representation of form.

Coherence thresholds for detecting concentric structure have been reported to be lower than those for detecting parallel structure (Dakin 1997a, 1999; Wilson et al. 1997; Wilson and Wilkinson 1998, 2003; Kurki and Saarinen 2004; Lewis et al. 2004). There is also evidence that the processing of concentric and parallel structure requires different degrees of spatial integration (Wilson et al. 1997; Wilson and Wilkinson 1998; Braddick et al. 1999). However, our research has recently shown a spatio-temporal interaction in the processing of these forms that leads to optimal sensitivity occurring for different combinations of stimulus duration and spatial extent for these two types of global forms (Aspell et al. 2006).

To further investigate the mechanisms that underlie the integration of local information into global forms, and in particular, to determine whether different human brain areas are activated by concentric and parallel forms, we used fMRI to compare brain activation to concentric and parallel forms constructed from oriented arrays of short line elements.

Because vertical and horizontal contours have different environmental significance (Switkes et al. 1978; Keil and Cristóbal 2000) and show differences in visual processing (Li et al. 2003; (Romani et al. 2003), parallel textures in these two orientations were tested separately, and the comparison between them provided one of the significant outcomes of this study.

\section{Methods}

Six healthy subjects (two males and four females, aged 1929 years) with normal or corrected-to-normal vision gave their informed written consent to participate in the study. All experiments followed protocols approved by the Oxford Research Ethics Committee.

\section{Stimuli}

All stimuli were back-projected using an XGA projector (Sanyo, Watford, UK) onto a white projection screen, positioned at a viewing distance of $244 \mathrm{~cm}$. Subjects lay supine in the scanner and viewed the screen using prism glasses (Wardray-Premise, Thames Ditton, UK).

\section{Global form stimuli}

The form stimuli were created using programs written in the Lua environment, version 5.0 (Ierusalimschy 2003). Arrays of 2,530 short black line elements were presented in 
a rectangular area $\left(19.8^{\circ} \times 19.8^{\circ}\right)$ against a white background. Concentric forms were created by orienting the line elements, in a $10^{\circ}$ region surrounding the central fixation square, tangentially to virtual concentric circles (see Fig. 1). Parallel forms were created by orienting the line elements in the central region either vertically or horizontally. The line elements outside the central form region were oriented randomly. A centrally located fixation square was present throughout the experiment.

The stimuli were presented in a block design, with separate 30 s blocks of: (1) concentric forms, (2) vertical parallel forms, (3) horizontal parallel forms and (4) random arrays of line elements. Within block types (1)-(3), the form stimulus alternated, every $\sim 2 \mathrm{~s}$ with a random array of line elements (the exact duration varied from a maximum of $2.5 \mathrm{~s}$ and a minimum of $1.5 \mathrm{~s}$ from trial to trial, in order to prevent the timing of change being predictable). In the random block (4) an unrelated random array was presented every $\sim 2 \mathrm{~s}$. Each run lasted $6 \mathrm{~min}$ and consisted of 12 blocks: three concentric, three vertical parallel, three horizontal parallel and three random, with the different block types presented in a random sequence. Subjects had a short rest between runs, and each subject completed five runs. Subjects were instructed to maintain fixation on the central small coloured square throughout. To maintain attention, they were required to press a button every time the square changed colour (a change that was not synchronous with the alternations between coherent form and random patterns).

Form region localiser

In the same session, to locate the region in each retinotopic area activated by the central form (as opposed to the random surround), we presented a circular flickering checkerboard that exactly matched the location and size of the central form region. Blocks of $15 \mathrm{~s}$ duration were presented, and checkerboard blocks alternated with blocks in which only the grey fixation screen was presented. No behavioural task was used.
FFA and LOC localisers

To localise two important object-selective areas, the fusiform face area (Kanwisher et al. 1997) and the lateral occipital complex (Malach et al. 1995), localiser scans were also carried out for each subject. The stimuli used were grey-scale photographs of faces, inanimate objects or textures. Images of faces were taken from a database of the Psychological Image Collection at Stirling (PICS: http:// www.pics.psych.stir.ac.uk/) and had not previously been seen by any of the subjects. Photographs of inanimate objects and textures were obtained from sources including CorelDraw and Microsoft clip-art. We used the Presentation $^{\circledR}$ software (http://www.neurobs.com) to control the delivery of these stimuli. Each stimulus type (face/object/ texture) was presented in separate $12 \mathrm{~s}$ blocks. Twelve images were presented in each stimulus block and each image was presented for $800 \mathrm{~ms}$, followed by a $200 \mathrm{~ms}$ grey fixation screen. Stimulus blocks were alternated with 'blank' blocks in which a grey fixation screen was present throughout. Subjects performed a one-back matching task using a response box. Each stimulus condition was repeated eight times in a counterbalanced block design.

\section{Retinotopic stimuli}

Standard retinotopic mapping (Warnking et al. 2002) was performed in an additional scanning session for each subject. The stimuli consisted of flickering black-and-white checks, reversing contrast at a frequency of $8 \mathrm{~Hz}$, that formed either a thin, eccentrically expanding ring (used for mapping eccentricity) or a $45^{\circ}$ pie wedge-shaped configuration that advanced, rotating in the clockwise direction, by $30^{\circ}$ every $4 \mathrm{~s}$ (i.e. every TR). The rotating wedge was used for mapping the angular dimension. The rotating wedge blocks, of duration $4.8 \mathrm{~min}$, alternated with $3.2 \mathrm{~min}$ expanding ring blocks. A total of six wedge blocks and four ring blocks were presented and subjects maintained central fixation throughout.
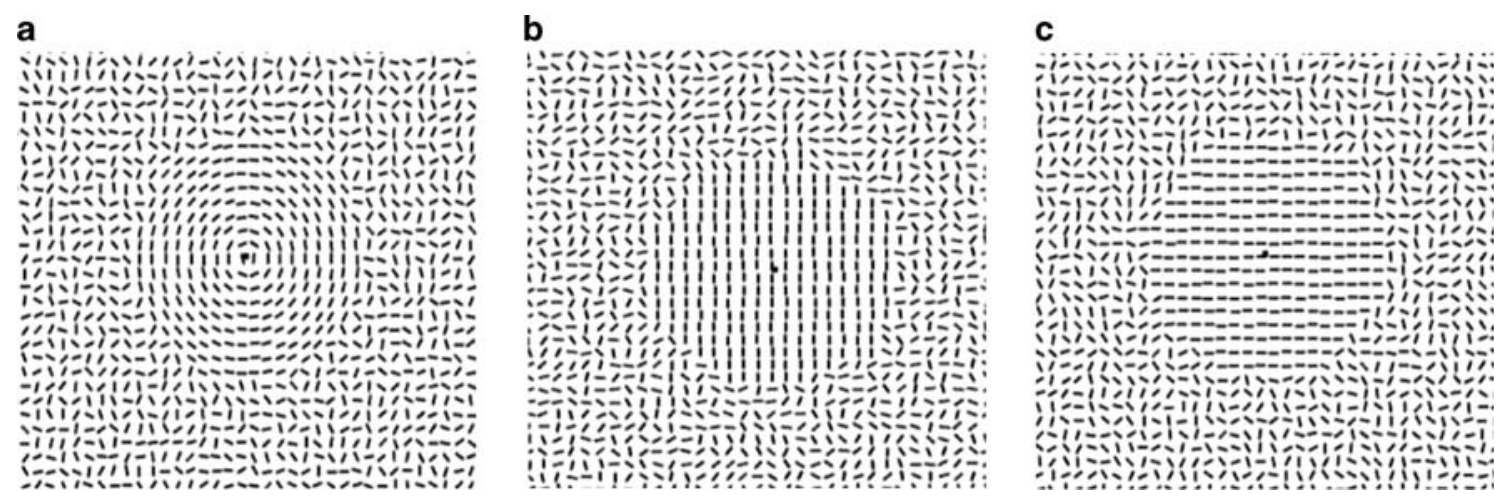

Fig. 1 Form coherence stimuli consisting of oriented line elements, a concentric, b vertical parallel, c horizontal parallel 


\section{fMRI methods}

\section{Imaging parameters}

The following parameters were used for the main form stimulus experiment, the form region localiser and the FFA/LOC localiser experiments. Magnetic resonance images were acquired using the Varian-Inova 3T scanner at the Centre for Functional Magnetic Resonance Imaging of the Brain (FMRIB) in Oxford, UK, with a head-dedicated gradient insert coil (Magnex, Oxford, UK). Functional imaging was performed using a gradient-echo EPI sequence (TR(repetition time) $3 \mathrm{~s}$, TE(echo time) $30 \mathrm{~ms}$, $192 \mathrm{~mm} \times 192 \mathrm{~mm}$ FOV, voxel size $3 \times 3 \times 3 \mathrm{~mm}$ ). Twenty-four $3 \mathrm{~mm}$ slices were acquired to cover a posterior section of the brain, encompassing all of the occipital lobe. An automated shimming algorithm was used to reduce magnetic field inhomogeneities. Whole brain T1 weighted anatomical images were also acquired for each subject, with a voxel size of $1 \times 1 \times 1 \mathrm{~mm}$.

\section{Retinotopy data collection}

Echo planar images (EPI), oriented perpendicular to the calcarine sulcus, were acquired with a quadrature surface coil (NOVA Medical, Wakefield, MA) covering the occipital pole, using typical parameters $(\mathrm{TR}=4 \mathrm{~s}, \mathrm{TE}=30 \mathrm{~ms}$, $128 \times 128 \mathrm{~mm}$, voxel size $2 \times 2 \times 2 \mathrm{~mm}$ FOV, thirty-two $2 \mathrm{~mm}$ slices).

\section{Analysis}

Analysis of the main form stimulus experiment and the form region localiser experiment was carried out using FEAT (FMRI Expert Analysis Tool) Version 5.4, part of FSL (FMRIB's Software Library, http://www.fmrib. ox.ac.uk/fsl). In the main form stimulus experiment the activation during the random array blocks served as the baseline, and in the form region localiser experiment, the activation during the grey fixation blocks served as the baseline. The first four volumes were discarded to allow for $\mathrm{T} 1$ equilibrium effects. The following pre-statistics processing was applied: motion correction using MCFLIRT (Jenkinson et al. 2002); non-brain removal using BET (Smith 2002); spatial smoothing using a Gaussian kernel of FWHM $5 \mathrm{~mm}$; mean-based intensity normalisation of all volumes by the same factor and highpass temporal filtering. Time-series statistical analysis was carried out using FILM with local autocorrelation correction (Woolrich et al. 2001). $Z$ (Gaussianised T/F) statistic images were thresholded using clusters determined by $Z>2.3$ and a (corrected) cluster significance threshold of $p=0.05$ (Worsley et al. 1992).
Functional images were registered to the subject's anatomical scan and then to the Montreal Neurological Institute 152-mean brain using FLIRT (Jenkinson and Smith 2001; Jenkinson et al. 2002). The fMRI response (percent signal change relative to the random blocks) was calculated separately for each subject for each retinotopic area and the FFA and LOC regions of interest (see below) using the FSL program Featquery.

The fusiform face area (FFA) regions of interest (ROIs) were created by locating voxels in the fusiform gyrus that responded significantly more to faces than to inanimate objects (face, object contrast). Lateral occipital complex (LOC) ROIs were created from an object, face contrast. In each subject, a region of the fusiform gyrus showed significant activation in the face, object contrast. The Talairach co-ordinates of this region were consistent with those previously reported for the FFA (Kanwisher et al. 1997). A more posterior region on the lateral surface of the occipital lobe was found in the object, face contrast. We note that in many previous studies LOC is localized by the contrast intact objects $>$ scrambled objects or textures. Our objects $>$ faces contrast located a region with co-ordinates that correspond well to regions previously described as the LOC (GrillSpector et al. 1999) but we note that we did not use a standard method to localise this region.

Cortical surface reconstruction, inflation and flattening were carried out using the Freesurfer program package (Dale et al. 1999; Fischl et al. 1999), and see http:// surfer.nmr.mgh.harvard.edu/). Mapping of the borders between the retinotopic visual areas was conducted using FsFast, part of Freesurfer. Raw images were first motioncorrected, and then intensity-normalized using the average in brain voxel intensity. A fast Fourier analysis was conducted on the time-series of each voxel to statistically correlate retinotopic stimulus location with cortical anatomy. The phase component of the signal was used to code retinotopic location. After registration of the functional and anatomical images, the functional data could be viewed on the inflated and/or flattened cortical surface.

The data from the expanding ring and rotating wedge scans were combined to yield field sign maps (mirrorimage vs. non-mirror-image visual field representation). This method automatically and objectively defines visual borders because adjacent early visual areas have opposite field signs (see Sereno et al. 1993 for details). For each subject, the Freesurfer program was used to draw the outline of areas $\mathrm{V} 1, \mathrm{~V} 2, \mathrm{VP}, \mathrm{V} 3, \mathrm{~V} 3 \mathrm{~A}$, and $\mathrm{V} 4 \mathrm{v}$ in each hemisphere. These areas have all been previously described (e.g. Tootell et al. 1997). We did not attempt to identify retinotopic areas in more anterior regions.

To specifically measure percent signal change that could be attributed to the central form region (as opposed to the random surround), 'form region masks' were created by 
Fig. 2 fMRI activation to concentric (upper panels), horizontal (middle panels) and vertical (lower panels) forms, overlaid on flattened occipital cortex from two subjects. $L$ indicates left hemisphere cortex, $R$ indicates right hemisphere cortex and labels indicate retinotopic areas $\mathrm{V} 1, \mathrm{~V} 2, \mathrm{~V} 3, \mathrm{~V} 3 \mathrm{~A}, \mathrm{~V} 4$ with corresponding borders

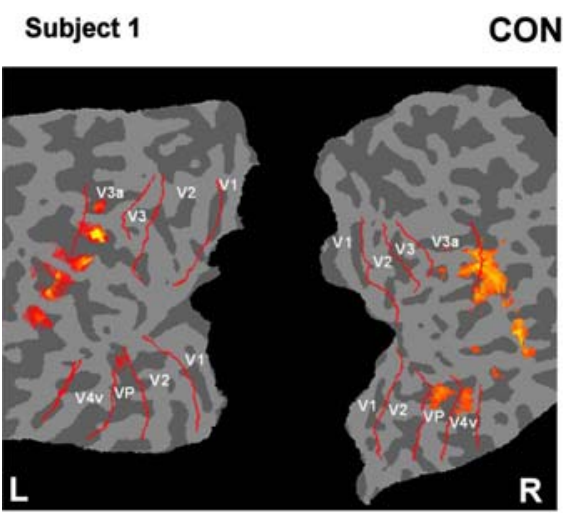

CONCENTRIC Subject 2

$\mathbf{R}$

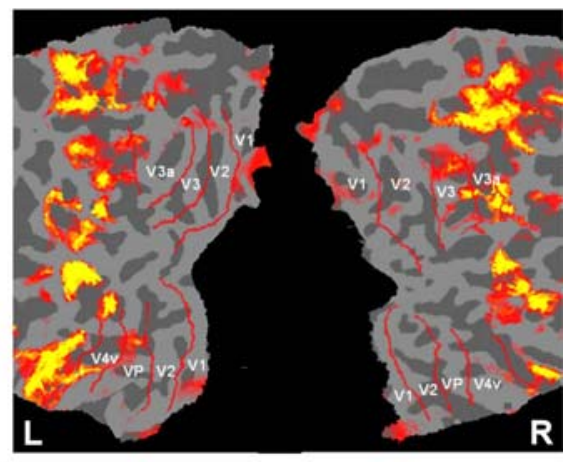

HORIZONTAL
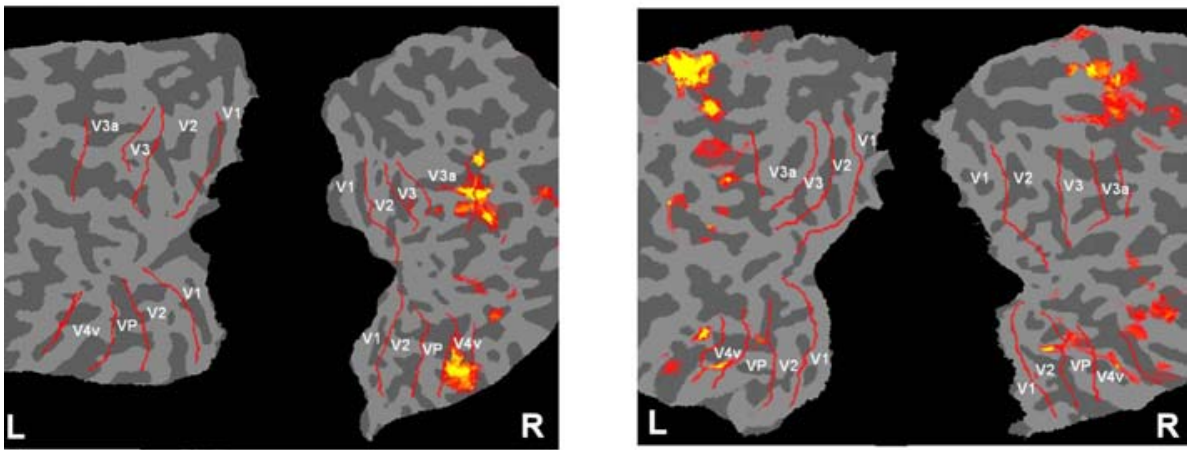

VERTICAL

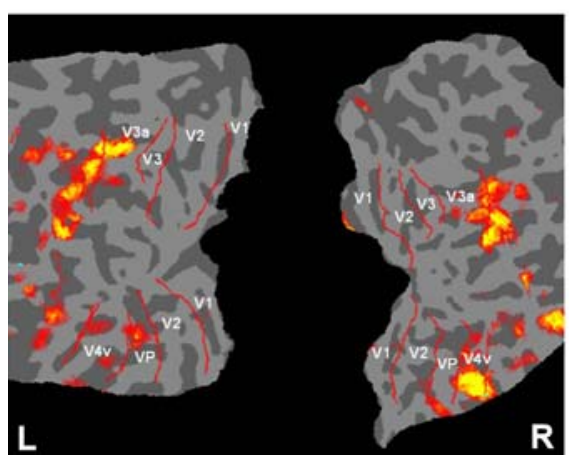

identifying voxels within each retinotopic area that were activated by the form region localiser checkerboard. To measure percent signal change at the horizontal and vertical meridian representations, we calculated percent signal change in the sub-regions that corresponded retinotopically to the central form region at the V1/V2, V3/V3A and VP/ $\mathrm{V} 4$ borders (the representations of the vertical meridian) and at the V1 midline and the V2/V3 border (the representations of the horizontal meridian).

\section{Results}

We compared the activation (percent signal change relative to random blocks) in the retinotopic areas V1, V2, V3, V3A and V4 and in the FFA and the LOC by concentric forms, vertical parallel forms and horizontal parallel forms. Figure 2 shows the fMRI activation to concentric, horizontal and vertical forms overlaid on flattened occipital cortex for two example subjects. Figure 3 plots the percentage signal change (average data for all six subjects) in the retinotopic regions corresponding to the central form region within each ROI for the different stimulus types. From the figure it can be seen that levels of brain activation vary depending on the stimulus presented, and overall that vertical patterns produced the highest percentage signal change. It is also evident that the differential effect of each stimulus type varied for different brain regions (ROIs).

A $3 \times 7$ repeated measures ANOVA with factors stimulus type (concentric/horizontal/vertical) and ROI (V1/V2/ $\mathrm{V} 3 / \mathrm{V} 3 \mathrm{~A} / \mathrm{V} 4 / \mathrm{FFA} / \mathrm{LOC}$ ) revealed a significant main effect of stimulus type, $F(2,10)=8.03 ; p=0.008$; a significant 
Fig. 3 fMRI activation (percentage signal change relative to baseline) to concentric, horizontal and vertical forms in the retinotopic regions corresponding to the central form region within each in cortical areas $\mathrm{V} 1, \mathrm{~V} 2, \mathrm{~V} 3$, V3A, V4, LOC and FFA. Error bars represent standard errors of the mean across subjects

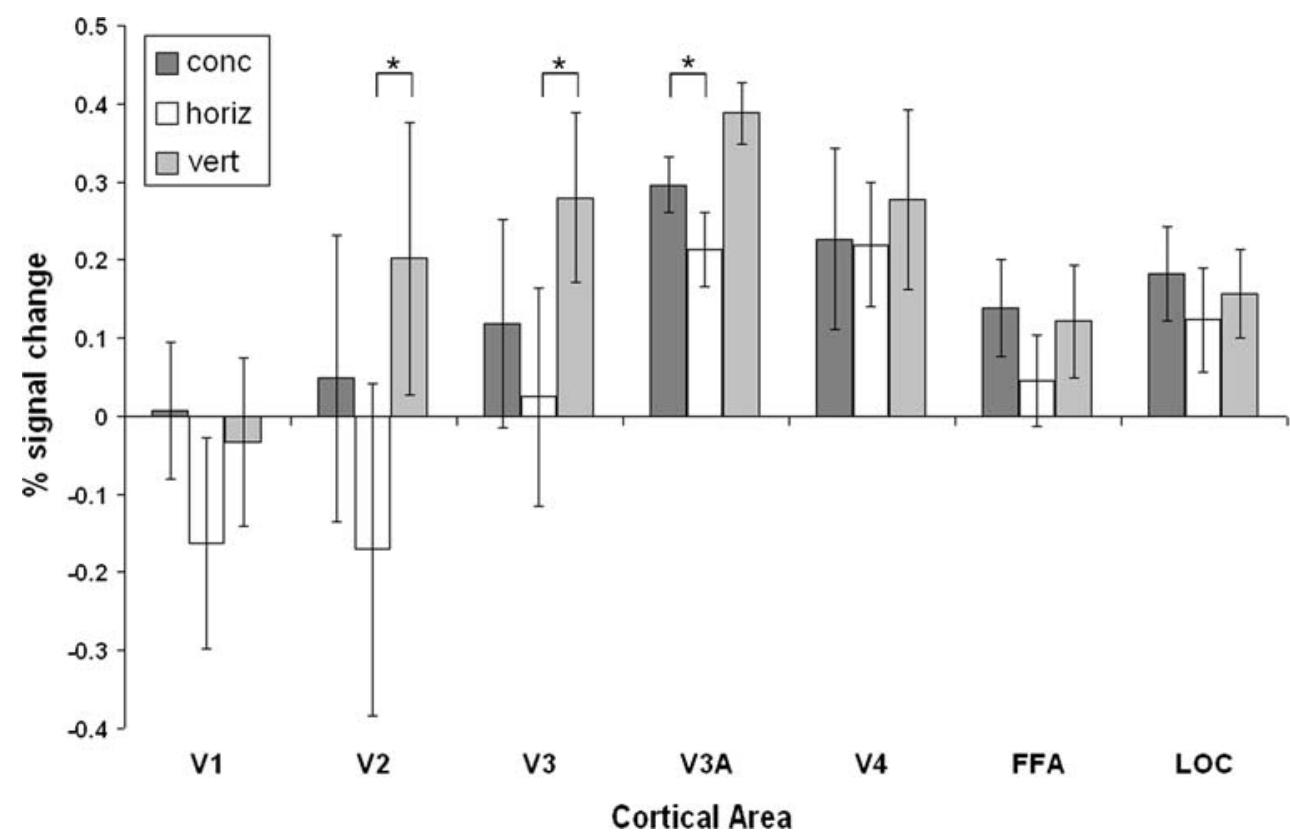

Table $1 p$ Values and $F$ values for repeated measures ANOVA and $p$ values for post hoc Bonferroni test

\begin{tabular}{|c|c|c|c|c|c|}
\hline \multicolumn{3}{|c|}{ ANOVA } & \multirow{2}{*}{\multicolumn{3}{|c|}{$\begin{array}{l}\text { Post hoc test (Bonferroni) } \\
p \text { value }\end{array}$}} \\
\hline \multirow[t]{2}{*}{ ROI } & \multirow[t]{2}{*}{$p$ value } & \multirow[t]{2}{*}{$F$ value } & & & \\
\hline & & & $\mathrm{H}-\mathrm{C}$ & $\mathrm{V}-\mathrm{C}$ & $\mathrm{H}-\mathrm{V}$ \\
\hline V1 & 0.167 & 2.153 & 0.229 & 1.000 & 0.883 \\
\hline $\mathrm{V} 2$ & $0.002 *$ & 12.032 & 0.071 & 0.166 & $0.033^{*}$ \\
\hline V3 & $0.008^{*}$ & 8.158 & 0.676 & 0.154 & $0.025^{*}$ \\
\hline V3A & $0.030^{*}$ & 4.868 & $0.044^{*}$ & 0.726 & 0.863 \\
\hline V4 & 0.466 & 0.824 & 1.000 & 0.937 & 0.120 \\
\hline LOC & 0.685 & 0.393 & 1.000 & 1.000 & 1.000 \\
\hline FFA & 0.146 & 2.342 & 0.200 & 1.000 & 0.433 \\
\hline
\end{tabular}

main effect of ROI, $F(6,30)=2.44 ; p=0.049$, and a significant interaction between stimulus type and ROI, $F(12,60)=2.95 ; p=0.003$. Significant differences between means (tested with the Bonferroni post hoc test) were found between vertical and horizontal stimulus types $(p=0.042)$, but not between vertical and concentric $(p=0.708)$ nor between horizontal and concentric $(p=0.116)$. Given the significant interaction between stimulus type and ROI in the first ANOVA, we ran separate ANOVAs for each ROI to compare the activation by different stimulus types. Table 1 shows the statistical results for all 7 ROIs. V2, V3 and V3A showed significant $(p<0.05)$ main effects of stimulus type, and post hoc tests revealed significant differences between horizontal and vertical stimulus types for V2 and V3, and a significant difference between concentric and horizontal for V3A. For all other ROIs there were no significant effects of stimulus type.
In order to rule out the possibility that VI is also differentially activated by vertical and horizontal stimuli we ran a $2 \times 3$ repeated measures ANOVA with factors stimulus type (horizontal, vertical) and ROI (V1, V2, V3). This revealed a significant effect of stimulus type $\left(F_{2,5}=8.70\right.$; $p=0.032), \quad$ a significant effect of ROI $\left(F_{2,5}=4.17\right.$; $p=0.017)$ and, importantly, a significant interaction between stimulus type and ROI $\left(F_{2,10}=10.20 ; p=0.004\right)$.

One possible source of the horizontal-vertical difference could be the suggested difference between radially and tangentially oriented contours (relative to the fovea) (Sasaki et al. 2006). To examine the influence of such an effect on our data, we compared the activation to the different stimuli in the ROIs corresponding to the horizontal meridian (where vertical lines are tangential and horizontal lines radial) and in the ROIs corresponding to the vertical meridian (where the geometrical relation is reversed). This analysis did not reveal any consistent differences (Fig. 4), although overall the brain activation to horizontal stimuli was lowest, as for the other ROIs. A $3 \times 2$ repeated measures ANOVA with factors stimulus type (concentric/horizontal/vertical) and ROI (horizontal meridian/vertical meridan) found no significant main effects and no significant interactions $(p>0.05)$.

\section{Discussion}

The present study used fMRI to compare the brain activation to concentric and parallel patterns. Our main, and unexpected, finding is that vertical and horizontal parallel patterns differentially activated visual cortex. Brain activations to vertical pattern stimuli were significantly higher 
Fig. 4 fMRI activation (percentage signal change relative to baseline) to concentric, horizontal and vertical forms in the retinotopic representation of the vertical meridian (top panel) and horizontal meridian (bottom panel). Error bars represent standard errors

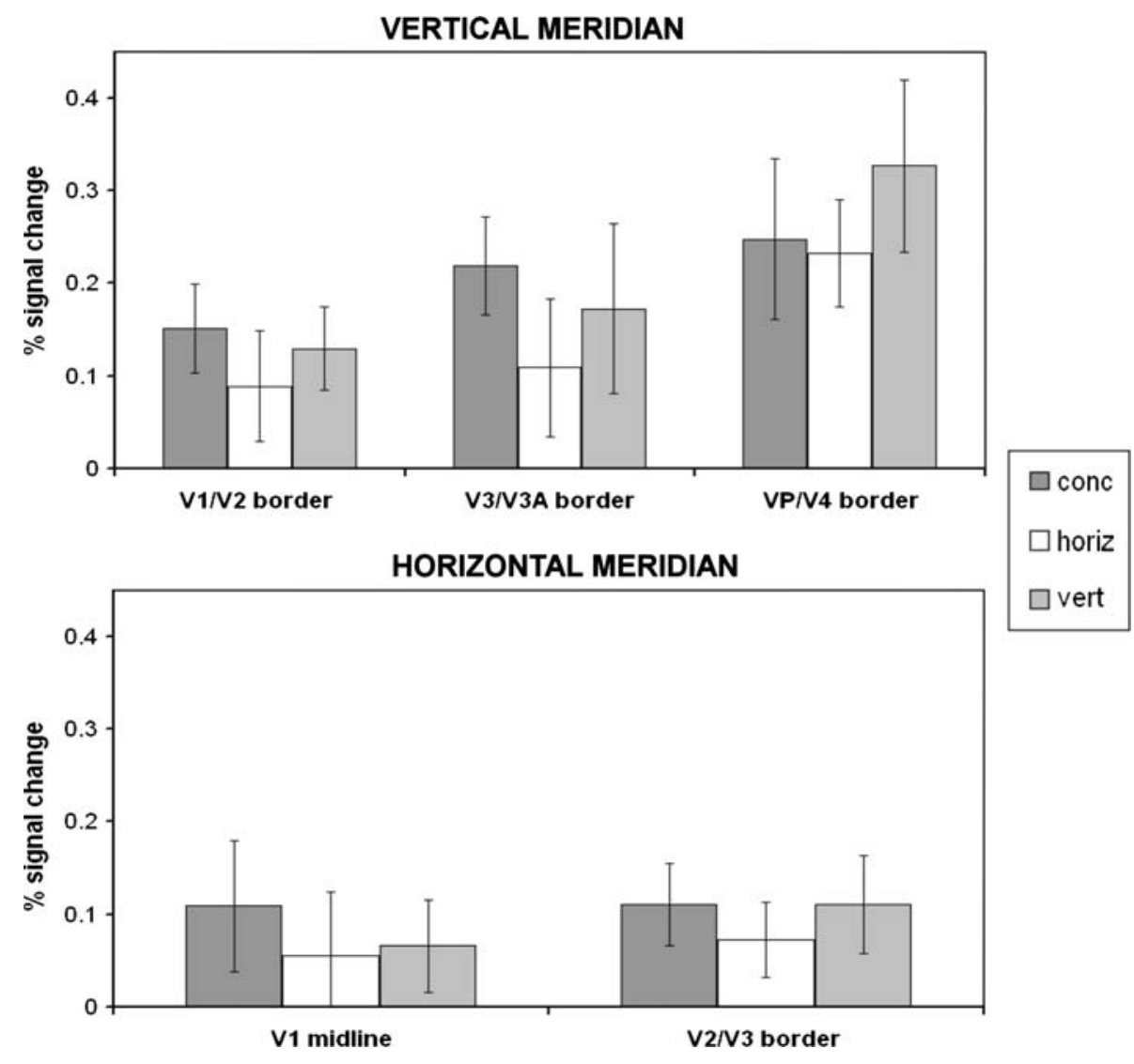

than those to horizontal pattern stimuli in areas V2 and V3. However, in general, activations to concentric and parallel forms did not differ. The data suggest that the differential brain activation by the two orientations is due to differences in global (rather than local) visual processing since this differential activity was not found in V1 and the contrast with a random array pattern baseline was designed to specifically isolate differences in global processing. Only area V3A showed a significant difference between concentric and parallel (horizontal) stimuli, with the former stimulus better activating this area.

The relative degree of activation to the different form types varied according to brain area, but most showed the general trend of activation (from highest to lowest) in the order: vertical $>$ concentric $>$ horizontal. In V1, levels of activation by these stimulus types were not significantly different from each other and showed no effect of the coherent versus random contrast. This finding supports the idea that the effects found in higher visual areas were due to the global organization of the patterns, whereas V1 activity was primarily driven by local stimulus properties which do not differ systematically between coherent and random patterns. It also implies that the differential effects seen in the higher, extrastriate, areas arise from processing in these areas rather than from properties inherited in their input from V1. We note that V1 may have shown reduced activation in the form conditions compared to the random condition because of feedback from higher visual areas. Murray et al. (2002) found that when line elements were grouped into objects, activity increased in area LOC but decreased in V1. They interpreted the activity decrease in V1 as a consequence of feedback from higher visual areas when the objects were detected. In this way, activity in higher visual areas may 'explain away' the activity in lower visual areas since the need for the latter to signal the presence of the local elements is reduced once they have been grouped by the higher areas.

In the higher visual cortical areas, the global form stimuli generally produced higher activation than did the random arrays. This finding is consistent with a recent fMRI study (Dumoulin and Hess 2007)_using concentric and random 'flow field' Gabor array stimuli-which found higher activation to concentric forms in V4 and V3/VP. It is also consistent with single unit studies showing stronger neural responses to concentric forms in macaque V4 (Gallant et al. 1993). The present data further reveal that vertical global patterns better activate V2 and V3 than do horizontal global patterns, and concentric patterns activate V3A better than do horizontal forms. The other comparisons for these areas and all other comparisons for other areas (V1, V4, FFA, LOC) did not reach significance.

What can explain the unexpected finding of a difference between horizontal and vertical patterns? Previous 
observations (Furmanski and Engel 2000; Li et al. 2003; Wang et al. 2003; Xu et al. 2006) have revealed that there is more 'neural machinery' devoted to processing the cardinal (horizontal and vertical) orientations than the oblique orientations; these results have been related to the greater psychophysical sensitivity to cardinal than oblique orientations ('the oblique effect'; Appelle 1972).The higher activation to vertical than horizontal patterns might be taken to similarly reflect an imbalance in the number of neurons that prefer each orientation. However, a single unit study in cat V1 (Li et al. 2003) found a greater abundance of neurons tuned to horizontal than to vertical orientations and studies in ferret V1 have shown that larger cortical areas are devoted to horizontal than to vertical orientations (Chapman and Bonhoeffer 1998; Coppola et al. 1998). In contrast, a recent study using high-field fMRI in humans (Yacoub et al. 2008) found that there was a bias towards vertical stimuli (with horizontal motion) among orientation-selective columns in V1 (although only a subsection of V1 was analysed in this study).

With regards to perceptual differences, greater sensitivity to vertical than to horizontal stimuli has been found with broad-band stimuli (Essock et al. 2003; Hansen and Essock 2004). Essock et al. suggest that this 'horizontal effect' might act to minimize the perceptual saliency of the horizontal content that often predominates in natural scenes, thereby enhancing the relative salience of objects made up of a range of orientations. We investigated thresholds for vertical and horizontal form stimuli (using identical stimuli to those used in the fMRI experiment) on two subjects. The psychophysical task was a two-interval forced choice and subjects had to indicate in which of the two intervals they saw a form appear. A Bayesian adaptive method was used to sample a range of coherence levels and converged on the level at which subjects gave $75 \%$ correct performance on the task. Mean thresholds were derived from the average of five staircase runs. (Further stimulus details are in Aspell et al. 2006.) No significant differences $(p>0.05)$ between vertical and horizontal thresholds were found for either subject (in contrast to the findings of Essock et al.), but we note that more subjects should be tested in order to make firm conclusions regarding differential sensitivity to these stimuli.

The relationship between brain activity, numbers of preferentially tuned neurons and perceptual sensitivity is clearly a complex one, and may depend on the type of stimulus and may possibly vary between species. It is notable that most published neural data relate to primary visual cortex; our results suggest that any orientation biases at this level are not necessarily inherited by higher visual areas.

An alternative explanation for the difference in response to vertical and horizontal patterns is that it arises from the 'radial orientation bias' proposed by Tootell et al. (Sasaki et al. 2006). This predicts (and their fMRI study supported) that vertical orientations that are collinear with the centre of gaze will better activate the representation of the vertical meridian, and horizontal orientations collinear with the centre of gaze will better activate the representation of the horizontal meridian. We used our data to test this hypothesis by comparing the activation in the vertical meridian (V1/ V2 border, V3/V3A border and VP/V4 border) and horizontal meridian (V1 midline and V2/V3 border) to the vertical and horizontal (and concentric) forms. We did not find any interaction between the degree of activation to each stimulus type and the meridian representation (horizontal or vertical) ROIs, and so our data do not provide supporting evidence for the radial bias hypothesis. However, our stimuli were considerably different from the contrast reversing, phase-shifting gratings used by Sasaki et al. (2006), which were compared with baseline responses to a uniform grey display. Our comparison of oriented texture with random elements was designed to emphasise global processing. Such global processing may show quite different orientation anisotropies compared to the contrast detection tested by Sasaki et al.'s comparison.

It is worth noting that a potential reason for the existence of a vertical bias independent of field location is that vertical contours, but not horizontal, are informative about binocular disparity. To test whether this is relevant to the differences we observed, it would be interesting to test these stimuli with monocular as well as binocular presentation and investigate the level of binocular interaction for the two orientations. However, it should be noted that disparity tuning of cortical neurons is not necessarily correlated with their preferred contour orientation (Cumming 2002).

We found that concentric forms produced greater activation in only one area, V3A, where concentric activation was higher than horizontal. The homology between macaques and humans is problematic for V3A (Vanduffel et al. 2001) so it is difficult to make comparisons with data from monkeys. Human V3A has mainly been investigated with respect to its motion sensitivity see e.g. (Tootell et al. 1997; Braddick et al. 2000; Liu et al. 2004; Aspell et al. 2005; Koyama et al. 2005); however, it is also known to be important in form processing (Schira et al. 2004). Neurons in V3A have relatively large receptive fields and contain a complete, contiguous representation of the visual field, making them potentially well-suited for an involvement in form integration. Previous studies suggested that concentric structure is processed by different mechanisms than is parallel structure (Wilson et al. 1997; Wilson and Wilkinson 1998; Wilkinson et al. 2000; Kurki and Saarinen 2004), in particular, for concentric stimuli, the local orientation information is combined over larger spatial extents (Wilson et al. 1997; Aspell et al. 2006). Given this, it is likely that concentric structure would be efficiently processed by 
neurons with large receptive fields, such as those in V3A. The reason for the difference between concentric and horizontal, but not between concentric and vertical in V3A is unclear. In any case, the relation between form processing and motion processing in area V3A deserves further investigation.

Our findings can be compared to those of Wilkinson et al. (2000) who reported that V4 and FFA were better activated by concentric gratings than by parallel gratings. Although we found a difference in V3A, we did not find a significant difference between concentric and horizontal or vertical parallel forms in V4 or FFA. However, there were some major differences between the stimuli used in these studies: Wilkinson et al. (2000) used vertical parallel gratings and their stimuli were composed of black and white gratings, not oriented line elements as in the present study. In addition, in the Wilkinson et al. (2000) study, so that subjects could maintain attention, the concentric forms slightly changed shape and the parallel forms slightly changed orientation every few seconds, whereas in the present study the concentric/parallel forms alternated with random arrays of line elements every 2 -s. This latter difference could have had a significant impact on the level of brain activity measured over a block. As discussed above with respect to Sasaki et al. (2006), the present study also differs in the 'baseline stimulus' used: in the present study, we used randomly oriented elements rather than the blank screen of mean luminance used by Wilkinson et al. (2000) which we argue will reflect responses to local as well as global structure.

We reasoned that using a random texture baseline ought to better isolate the processing involved in the integration of local elements into a global form. A baseline consisting of a uniform grey screen (as in Wilkinson et al. 2000; Sasaki et al. 2006) is more likely to isolate differences at local levels than is our random array baseline and it may be that the horizontal-vertical difference that we find only arises at global/intermediate levels of representation, not at more local levels. The fact that we find no difference in activation to the horizontal and vertical pattern stimuli in area $\mathrm{V} 1$ is compatible with this interpretation.

In conclusion, we find that horizontal and vertical parallel forms made up of short oriented line elements differentially activate human extrastriate visual cortical areas, with vertical forms producing an overall greater activation. Our data suggest that this difference in brain activation to vertical and horizontal form stimuli is due to differences at global or intermediate levels of pattern representation, since V1 did not show differential activity for these stimuli, whereas mid-level retinotopic areas (V2 and V3) did. The robustness and generality of this surprising finding should be tested in future studies with different types of form stimuli, e.g. gratings, Gabor arrays and Glass patterns, and also with different imaging methods, e.g. electroencephalography (EEG). This work will be important to understand the basis of this finding and how it relates to the complex but under-studied mechanisms of mid-level vision.

Acknowledgments This work was supported by Medical Research Council (UK) Programme Grant \# G7908507. Thanks to H. Bridge for her help with the retinotopy analyses.

\section{References}

Achtman RL, Hess RF, Wang Y-Z (2003) Sensitivity for global shape detection. J Vis 3:616-624

Allman J, Miezin F, McGuinness E (1985) Stimulus specific responses from beyond the classical receptive field: neurophysiological mechanisms for local-global comparisons in visual neurons. Annu Rev Neurosci 8:407-430

Appelle S (1972) Perception and discrimination as a function of stimulus orientation—oblique effect in man and animals. Psychol Bull $78: 266$

Aspell JE, Tanskanen T, Hurlbert AC (2005) Neuromagnetic correlates of visual motion coherence. Eur J Neurosci 22:29372945

Aspell JE, Wattam-Bell J, Braddick O (2006) Interaction of spatial and temporal integration in global form processing. Vis Res 46:28342841

Badcock D, Clifford C (2006) The inputs to global form. In: Jenkins M, Harris R (eds) Seeing spatial form. Oxford University Press, Oxford, pp 37-50

Bell J, Badcock DR, Wilson H, Wilkinson F (2007) Detection of shape in radial frequency contours: independence of local and global form information. Vis Res 47:1518-1522

Braddick OJ, Lin MH, Atkinson J, O'Brien J, Wattam-Bell J, Turner R (1999) Form coherence: a measure of extrastriate pattern processing. Perception 28:59

Braddick OJ, O'Brien JMD, Wattam-Bell J, Atkinson J, Turner R (2000) Form and motion coherence activate independent, but not dorsal/ventral segregated, networks in the human brain. Curr Biol 10:731-734

Chapman B, Bonhoeffer T (1998) Overrepresentation of horizontal and vertical orientation preferences in developing ferret area 17. Proc Natl Acad Sci USA 95:2609-2614

Coppola DM, White LE, Fitzpatrick D, Purves D (1998) Unequal representation of cardinal and oblique contours in ferret visual cortex. Proc Natl Acad Sci USA 95:2621-2623

Cumming BG (2002) An unexpected specialization for horizontal disparity in primate primary visual cortex. Nature 418:633-636

Dakin SC (1997a) The detection of structure in glass patterns: Psychophysics and computational models. Vis Res 37:2227-2246

Dakin SC (1997b) Glass patterns: some contrast effects re-evaluated. Perception 26:253-268

Dakin SC (1999) Orientation variance as a quantifier of structure in texture. Spat Vis 12:1-30

Dale AM, Fischl B, Sereno MI (1999) Cortical surface-based analysis I: segmentation and surface reconstruction. NeuroImage 9:179_ 194

Dumoulin SO, Hess RF (2007) Cortical specialization for concentric shape processing. Vis Res 47:1608-1613

Essock EA, DeFord JK, Hansen BC, Sinai MJ (2003) Oblique stimuli are seen best (not worst!) in naturalistic broad-band stimuli: a horizontal effect. Vis Res 43:1329-1335

Felleman DJ, Van Essen DC (1991) Distributed hierarchical processing in the primate cerebral cortex. Cereb Cortex 1:1-a-47 
Fischl B, Sereno MI, Dale AM (1999) Cortical surface-based analysis II: inflation, flattening, and a surface-based coordinate system. NeuroImage 9:195-207

Furmanski CS, Engel SA (2000) An oblique effect in human primary visual cortex. Nat Neurosci 3:535-536

Gallant JL, Braun J, Van Essen DC (1993) Selectivity for polar, hyperbolic, and cartesian gratings in macaque visual cortex. Science 259:100-103

Gallant JL, Shoup RE, Mazer JA (2000) A human extrastriate area functionally homologous to macaque V4. Neuron 27:227-235

Grill-Spector K, Malach R (2004) The Human Visual Cortex. Annu Rev Neurosci 27:649-677

Grill-Spector K, Kushnir T, Edelman S, Avidan G, Itzchak Y, Malach R (1999) Differential processing of objects under various viewing conditions in the human lateral occipital complex. Neuron 24:187-203

Hansen BC, Essock EA (2004) A horizontal bias in human visual processing of orientation and its correspondence to the structural components of natural scenes. J Vis 4(12):5, 1044-1060

Hubel DH, Wiesel TN (1968) Receptive fields and functional architecture of monkey striate cortex. J Physiol 195:215-243

Humphreys GW, Forde EM (2001) Hierarchies, similarity, and interactivity in object recognition: "category-specific" neuropsychological deficits. Behav Brain Sci 24:453-476

Ierusalimschy R (2003) Programming in Lua. Lua.org, Rio de Janerio

Jenkinson M, Smith SM (2001) A global optimisation method for robust affine registration of brain images. Med Image Anal 5:143-156

Jenkinson M, Bannister P, Brady M, Smith S (2002) Improved optimisation for the robust and accurate linear registration and motion correction of brain images. NeuroImage 17:825-841

Kanwisher N, McDermott J, Chun MM (1997) The fusiform face area: a module in human extrastriate cortex specialized for face perception. J Neurosci 17:4302-4311

Keil MS, Cristóbal G (2000) Separating the chaff from the wheat: possible origins of the oblique effect. J Opt Soc Am A 17:697-710

Kobatake E, Tanaka K (1994) Neuronal selectivities to complex object features in the ventral visual pathway of the macaque cerebral cortex. J Neurophysiol 71:856-867

Kofka K (1935) Principles of Gestalt psychology. Harcourt, New York

Kourtzi Z, Tolias AS, Altmann CF, Augath M, Logothetis NK (2003) Integration of local features into global shapes: monkey and human fMRI studies. Neuron 37:333-346

Kovacs I, Julesz B (1993) A closed curve is much more than an incomplete one: Effect of closure in figure-ground segmentation. Proc Natl Acad Sci USA 90:7495-7497

Koyama S, Sasaki Y, Andersen GJ, Tootell RBH, Matsuura M, Watanabe T (2005) Separate processing of different globalmotion structures in visual cortex is revealed by fMRI. Curr Biol 15:2027-2032

Kurki I, Saarinen J (2004) Shape perception in human vision: specialized detectors for concentric spatial structures? Neurosci Lett 360:100-102

Lamme VAF, Supèr H, Spekreijse H (1998) Feedforward, horizontal, and feedback processing in the visual cortex. Curr Opin Neurobiol 8:529-535

Lewis TL, Ellemberg D, Maurer D, Dirks M, Wilkinson F, Wilson HR (2004) A window on the normal development of sensitivity to global form in glass patterns. Perception 33:409-418

Li B, Peterson MR, Freeman RD (2003) Oblique effect: a neural basis in the visual cortex. J Neurophysiol 90:204-217

Liu T, Slotnick SD, Yantis S (2004) Human MT+ mediates perceptual filling-in during apparent motion. NeuroImage 21:1772-1780

Mahon LE, De Valois RL (2001) Cartesian and non-Cartesian responses in LGN, V1, and V2 cells. Vis Neurosci 18:973-981
Malach R, Reppas JB, Benson RR, Kwong KK, Jiang H, Kennedy WA, Ledden PJ, Brady TJ, Rosen BR, Tootell RB (1995) Objectrelated activity revealed by functional magnetic resonance imaging in human occipital cortex. Proc Natl Acad Sci USA 92:8135-8139

Maloney RK, Mitchison GJ, Barlow HB (1987) Limit to the detection of glass patterns in the presence of noise. J Opt Soc Am Opt Image Sci Vis 4:2336-2341

Maunsell JHR, Newsome WT (1987) Visual processing in monkey extrastriate cortex. Annu Rev Neurosci 10:363-401

Murray SO, Kersten D, Olshausen BA, Schrater P, Woods DL (2002) Shape perception reduces activity in human primary visual cortex. Proc Natl Acad Sci USA 99:15164-15169

Ostwald D, Lam JM, Li S, Kourtzi Z (2008) Neural coding of global form in the human visual cortex. J Neurophysiol 99:2456-2469

Pei F, Pettet MW, Vildavski VY, Norcia AM (2005) Event-related potentials show configural specificity of global form processing. Neuroreport 16:1427-1430

Romani A, Callieco R, Tavazzi E, Cosi V (2003) The effects of collinearity and orientation on texture visual evoked potentials. Clin Neurophysiol 114:1021-1026

Sasaki Y, Rajimehr R, Kim BW, Ekstrom LB, Vanduffel W, Tootell RBH (2006) The radial bias: a different slant on visual orientation sensitivity in human and nonhuman primates. Neuron 51:661670

Schira MM, Fahle M, Donner TH, Kraft A, Brandt SA (2004) Differential contribution of early visual areas to the perceptual process of contour processing. J Neurophysiol 91:1716-1721

Sereno MI, Dale AM, Reppas JB (1993) Borders of multiple visual areas in humans revealed by functional magnetic resonance imaging. Science 268:889-893

Smith S (2002) Fast robust automated brain extraction. Hum Brain Mapp 17:143-155

Smith MA, Bair W, Movshon JA (2002) Signals in macaque striate cortical neurons that support the perception of glass patterns. J Neurosci 22:8334-8345

Switkes E, Mayer MJ, Sloan JA (1978) Spatial frequency analysis of the visual environment: Anisotropy and the carpentered environment hypothesis. Vis Res 18:1393-1399

Tootell RBH, Mendola JD, Hadjikhani NK, Ledden PJ, Liu AK, Reppas JB (1997) Functional analysis of V3A and related areas in human visual cortex. J Neurosci 17:7060-7078

Vanduffel W, Fize D, Mandeville JB, Nelissen K, Van Hecke P, Rosen BR, Tootell RBH, Orban GA (2001) Visual motion processing investigated using contrast agent-enhanced fMRI in awake behaving monkeys. Neuron 32:565-577

Wang G, Ding S, Yunokuchi K (2003) Representation of cardinal contour overlaps less with representation of nearby angles in cat visual cortex. J Neurophysiol 90:3912-3920

Warnking J, Dojat M, Guérin-Dugué A, Delon-Martin C, Olympieff S, Richard N, Chéhikian A, Segebarth C (2002) fMRI retinotopic mapping - step by step. NeuroImage 17:1665-1683

Wilkinson F, James TW, Wilson HR, Gati JS, Menon RS, Goodale MA (2000) An fMRI study of the selective activation of human extrastriate form vision areas by radial and concentric gratings. Curr Biol 10:1455-1458

Wilson HR, Wilkinson F (1998) Detection of global structure in Glass patterns: implications for form vision. Vis Res 38:2933-2947

Wilson HR, Wilkinson F (2003) Further evidence for global orientation processing in circular Glass patterns. Vis Res 43:563-564

Wilson HR, Wilkinson F, Asaad W (1997) Concentric orientation summation in human form vision. Vis Res 37:2325-2330

Woolrich MW, Ripley BD, Brady JM, Smith SM (2001) Temporal autocorrelation in univariate linear modelling of FMRI data. NeuroImage 14:1370-1386 
Worsley KJ, Evans AC, Marrett S, Neelin P (1992) A three-dimensional statistical analysis for CBF activation studies in human brain. J Cereb Blood Flow Metab 12:900-918

Xu X, Collins CE, Khaytin I, Kaas JH, Casagrande VA (2006) Unequal representation of cardinal vs. oblique orientations in the middle temporal visual area. Proc Natl Acad Sci USA 103:17490-17495

Yacoub E, Harel N, Km UÄŸurbil (2008) High-field fMRI unveils orientation columns in humans. Proc Natl Acad Sci USA 105:10607-10612 